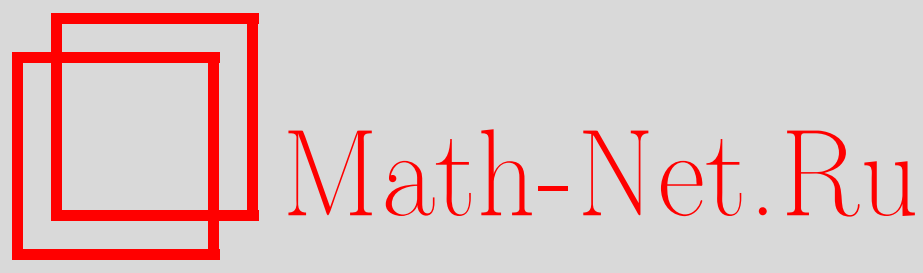

А. И. Зенчук, Многомерная линеаризуемая система уравнений типа $n$-волн, ТMФ, 2017, том 190 , номер $1,48-$ 57

DOI: https://doi.org/10.4213/tmf9205

Использование Общероссийского математического портала Math-Net.Ru подразумевает, что вы прочитали и согласны с пользовательским соглашением http://www.mathnet.ru/rus/agreement

Параметры загрузки:

IP : 54.224 .187 .69

26 апреля 2023 г., $07: 37: 42$

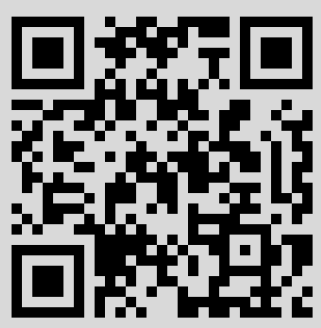




\title{
МНОГОМЕРНАЯ ЛИНЕАРИЗУЕМАЯ СИСТЕМА УРАВНЕНИЙ ТИПА $n$-ВОЛН
}

\begin{abstract}
Предложена линеаризуемая версия многомерной системы уравнений в частных производных типа $n$-волн. Система получена с помощью спектрального представления ее решений с использованием метода, аналогичного методу одевания для нелинейных уравнений в частных производных, интегрируемых методом обратной задачи рассеяния. Показана полная интегрируемость предложенной системы, а также построено ее частное решение.
\end{abstract}

Ключевые слова: уравнение $n$-волн, линеаризуемые уравнения, метод одевания, периодические решения.

DOI: $10.4213 / \operatorname{tmf} 9205$

\section{1. ВВЕДЕНИЕ}

Хорошо известное $(2+1)$-мерное уравнение $n$-волн [1], [2]

$$
\left[C^{(3)}, v_{t_{2}}\right]-\left[C^{(2)}, v_{t_{3}}\right]+C^{(2)} v_{t_{1}} C^{(3)}-C^{(3)} v_{t_{1}} C^{(2)}+\left[\left[C^{(3)}, v\right],\left[C^{(2)}, v\right]\right]=0
$$

где $C^{(i)}$ - постоянные диагональные матрицы, интегрируемое методом обратной задачи рассеяния (MO3P) [3]-[10], нашло широкое применение в математической физике. В частности, оно появляется в многомасштабных разложениях различных физических систем, где с помощью него описывается эволюция волновых пакетов. Уравнение (1) является бездиагональным $(N \times N)$-матричным уравнением $(N \geqslant 3)$ в частных производных первого порядка с квадратичной нелинейностью специального вида. Оно имеет $(N-1) N / 2 \geqslant 3$ различных производных $\sum_{i=1}^{3} V_{i}^{(\alpha \beta)} \partial_{t_{i}}$ по направлению вдоль векторов $V^{(\alpha \beta)}$ с координатами

$$
V_{1}^{(\alpha \beta)}=C_{\alpha}^{(2)} C_{\beta}^{(3)}-C_{\alpha}^{(3)} C_{\beta}^{(2)}, \quad V_{2}^{(\alpha \beta)}=C_{\alpha}^{(3)}-C_{\beta}^{(3)}, \quad V_{3}^{(\alpha \beta)}=C_{\beta}^{(2)}-C_{\alpha}^{(2)} .
$$

Работа выполнена при частичной поддержке РФФИ (грант № 14-01-00389) и Программы поддержки ведущих научных школ (грант НШ-9697.2016.2).

* Институт проблем химической физики РАН, Черноголовка, Московская обл., Россия. E-mail: zenchuk@itp.ac.ru 
Однако его естественное многомерное обобщение неинтегрируемо. Пример (частично) интегрируемого многомерного обобщения уравнения (1) имеет существенно измененную структуру. Одна из версий такого обобщения, обладающая решением в виде периодически расположенных лампов, построена в работе [11].

Обсуждаемое в настоящей работе нелинейное уравнение в частных производных в покомпонентной записи имеет вид

$$
\sum_{m=1}^{3} Z_{\alpha \beta}^{(m)}\left(v_{\alpha \beta}^{(l j)}\right)_{t_{m}}+\sum_{n=1}^{2} T_{\alpha \beta}^{(n)}\left(v_{\alpha \beta}^{(l j)}\right)_{x_{n}}-\sum_{k=1}^{2} \sum_{\gamma=1}^{N} a_{\alpha \gamma \beta}^{(k)}\left(v_{\alpha \gamma}^{(l k)} v_{\gamma \beta}^{(0 j)}-v_{\alpha \gamma}^{(l 0)} v_{\gamma \beta}^{(k j)}\right)=0,
$$

где $l, j=0,1,2, \alpha, \beta=1, \ldots, N$. Здесь $v^{(l j)}-(N \times N)$-матричные поля, а $Z_{\alpha \beta}^{(m)}$, $T_{\alpha \beta}^{(n)}, a_{\alpha \gamma \beta}^{(k)}-$ постоянные коэффициенты, имеющие специальный вид, заданный далее уравнениями (16)-(18). Структура уравнения (3) отличается от структуры $(2+1)$-мерного уравнения $n$-волн (1). В частности, в него входят шесть матричных полей $v^{(l j)}$ вместо одного. Подобно уравнению (1) уравнение (3) имеет квадратичную нелинейность и $N(N-1) / 2 \geqslant 3$ производных по направлению

$$
\sum_{m=1}^{3} Z_{\alpha \beta}^{(m)} \partial_{t_{i}}+\sum_{n=1}^{2} T_{\alpha \beta}^{(n)} \partial_{x_{n}}
$$

Поскольку наше пространство независимых переменных пятимерно, существует пять независимых производных по направлению.

Хотя структура уравнения (3) и допускает формальное отождествление различных полей $v^{(i j)}$ (точнее, возможна редукция $v^{(i j)}=C^{(i)} v^{(00)} C^{(j)}$ с некоторыми постоянными диагональными матрицами $\left.C^{(i)}, i=1,2\right)$, такая редукция кардинально "обрезает" пространство решений, уменьшая его размерность, так что редуцированная система теряет полную интегрируемость.

Характерной особенностью уравнения (3) является то, что ее линейный предел $\left(\right.$ при $\left.v^{(i j)} \rightarrow w^{(i j)}\right)$ имеет вид

$$
\sum_{m=1}^{3} Z_{\alpha \beta}^{(m)}\left(w_{\alpha \beta}^{(i j)}\right)_{t_{m}}+\sum_{n=1}^{2} T_{\alpha \beta}^{(n)}\left(w_{\alpha \beta}^{(i j)}\right)_{x_{n}}=0, \quad i, j=0,1,2, \quad \alpha, \beta=1, \ldots, N,
$$

одинаковый для всех $i, j$. Поскольку уравнение (3) линеаризуемо [12]-[14], его решения формально связаны с решениями соответствующей системы линейных уравнений в частных производных (4). В качестве вспомогательной техники при выводе этой связи используется спектральное представление решения и строится спектральная функция $\Psi(\lambda, \mu)$ двух спектральных параметров. Алгоритм построения полей $v^{(i j)}$ с помощью связанной с ними спектральной функции $\Psi$ будем называть методом одевания по аналогии с методом одевания для уравнений, интегрируемых с помощью МОЗР [6]-[9].

Статья имеет следующую структуру. В разделе 2 излагается частный метод одевания, используемый далее для вывода системы нелинейных уравнений в частных производных (3). Дополнительные алгебро-дифференциальные связи, накладываемые на поля этой системы, получены в разделе 3. Пространство решений для системы (3) обсуждается в разделе 4, где приводится пример частного решения. Основные результаты обсуждаются в разделе 5 . 


\section{2. МЕТОД ОДЕВАНИЯ}

2.1. Вывод спектрального уравнения. Рассмотрим $(N \times N)$-матричную функцию $R(x ; \lambda, \mu)$, зависящую от двух спектральных параметров $\lambda$ и $\mu$ и набора вспомогательных параметров $x=\left(x_{1}, x_{2}, \ldots\right)$, которые являются независимыми переменными нелинейных уравнений в частных производных:

$$
\begin{aligned}
R(\lambda, \mu ; x)= & \exp \left(\sum_{j=1}^{D} s^{(j)}(\lambda) x_{j}+\sum_{k} S^{(k)}(\lambda) t_{k}\right) R_{0}(\lambda, \mu) \times \\
& \times \exp \left(-\sum_{j=1}^{D} s^{(j)}(\mu) x_{j}-\sum_{k} S^{(k)}(\mu) t_{k}\right)+r(\lambda) q(\mu),
\end{aligned}
$$

где $r(\lambda)$ и $q(\mu)$ - некоторые произвольные диагональные функции спектральных параметров, $s^{(i)}$ - независимые произвольные диагональные функции спектрального параметра, а $S^{(m)}$ - линейные комбинации функций $s^{(i)}$,

$$
S^{(m)}(\lambda)=\sum_{i=1}^{D} A^{(m i)} s^{(i)}(\lambda), \quad m=1,2, \ldots,
$$

с постоянными диагональными матрицами $A^{(m i)}$. Матричная функция спектральных параметров $R_{0}$ в уравнении (5) такова, что оператор $R(\lambda, \mu)$ обратим. Функцию, обратную к $R$, будем называть спектральной функцией $\Psi$ :

$$
\int R(x, \lambda, \nu) \Psi(x ; \nu, \mu) d \Omega(\nu) \equiv R * \Psi=I \delta(\lambda-\mu),
$$

где $\Omega(\nu)$ - некоторая скалярная мера на плоскости комплексного спектрального параметра $\nu, I$ - единичная матрица размера $N \times N$. Дифференцируя функцию $\Psi$ по $x_{m}$ и учитывая ее определение $(7)$, а также выражение (5) для $R$, получаем формулы

$$
\begin{aligned}
\Psi_{x_{m}}(\lambda, \mu)= & -\Psi(\lambda, \mu) s^{(m)}(\mu)+s^{(m)}(\lambda) \Psi(\lambda, \mu)+ \\
& +\left(\Psi(\lambda, \nu) s^{(m)}(\nu)\right) * r(\nu) q(\tilde{\nu}) * \Psi(\tilde{\nu}, \mu)- \\
& -\Psi(\lambda, \nu) * r(\nu) q(\tilde{\nu}) *\left(s^{(m)}(\tilde{\nu}) \Psi(\tilde{\nu}, \mu)\right), \quad m=1, \ldots, D, \\
\Psi_{t_{m}}(\lambda, \mu)= & -\Psi(\lambda, \mu) S^{(m)}(\mu)+S^{(m)}(\lambda) \Psi(\lambda, \mu)+ \\
& +\left(\Psi(\lambda, \nu) S^{(m)}(\nu)\right) * r(\nu) q(\tilde{\nu}) * \Psi(\tilde{\nu}, \mu)- \\
& -\Psi(\lambda, \nu) * r(\nu) q(\tilde{\nu}) *\left(S^{(m)}(\tilde{\nu}) \Psi(\tilde{\nu}, \mu)\right), \quad m=1,2, \ldots .
\end{aligned}
$$

Уравнения (8), (9) можно считать системой уравнений для спектральной функции $\Psi$.

2.2. Вывод системы нелинейных уравнений в частных производных (3). Применяя теперь операторы $\left(q s^{(i)}\right) *$ и $*\left(s^{(j)} r\right), i=0,1,2\left(\right.$ где $\left.s^{(0)}=1\right)$, к уравнениям (8) и (9), приходим к системе нелинейных уравнений в частных производных для матричных полей

$$
v^{(i j)}=\left(q s^{(i)}\right) * \Psi *\left(s^{(j)} r\right),
$$




$$
\begin{gathered}
v^{(i n 0 j)}=\left(q s^{(i)} s^{(n)}\right) * \Psi *\left(s^{(j)} r\right), \quad v^{(i 0 n j)}=\left(q s^{(i)}\right) * \Psi *\left(s^{(n)} s^{(j)} r\right), \\
v^{(i 00 j)}=v^{(i 0 j 0)}=v^{(0 i 0 j)} \equiv v^{(i j)} .
\end{gathered}
$$

Эта система имеет следующий вид:

$$
\begin{aligned}
v_{x_{m}}^{(i j)} & +v^{(i 0 m j)}-v^{(i m 0 j)}-v^{(i m)} v^{(0 j)}+v^{(i 0)} v^{(m j)}=0 \\
v_{t_{m}}^{(i j)} & +\sum_{k=1}^{D}\left(v^{(i 0 k j)} A^{(m k)}-A^{(m k)} v^{(i k 0 j)}\right)- \\
& -\sum_{k=1}^{D} v^{(i k)} A^{(m k)} v^{(0 j)}+v^{(i 0)} \sum_{k=1}^{D} A^{(m k)} v^{(k j)}=0 .
\end{aligned}
$$

Используя соответствующую комбинацию уравнений (12), (13), можно исключить поля $v^{(i 0 m j)}$ и $v^{(i m 0 j)}$ и вывести систему для полей $(10)$. Например, полагая $D=2$, получаем (в покомпонентном виде) нелинейную систему

$$
\left|\begin{array}{ccccc}
\left(e_{t_{1}}^{(i j)}\right)_{\alpha \beta} & \left(e_{t_{2}}^{(i j)}\right)_{\alpha \beta} & \left(e_{t_{3}}^{(i j)}\right)_{\alpha \beta} & \left(e_{x_{1}}^{(i j)}\right)_{\alpha \beta} & \left(e_{x_{2}}^{(i j)}\right)_{\alpha \beta} \\
A_{\beta}^{(11)} & A_{\beta}^{(21)} & A_{\beta}^{(31)} & 1 & 0 \\
A_{\alpha}^{(11)} & A_{\alpha}^{(21)} & A_{\alpha}^{(31)} & 1 & 0 \\
A_{\beta}^{(12)} & A_{\beta}^{(22)} & A_{\beta}^{(32)} & 0 & 1 \\
A_{\alpha}^{(12)} & A_{\alpha}^{(22)} & A_{\alpha}^{(32)} & 0 & 1
\end{array}\right|=0
$$

где используются следующие обозначения:

$$
\begin{aligned}
& e_{x_{m}}^{(i j)}:=v_{x_{m}}^{(i j)}-v^{(i m)} v^{(0 j)}+v^{(i 0)} v^{(m j)} \\
& e_{t_{m}}^{(i j)}:=v_{t_{m}}^{(i j)}-\sum_{k=1}^{2} v^{(i k)} A^{(m k)} v^{(0 j)}+v^{(i 0)} \sum_{k=1}^{2} A^{(m k)} v^{(k j)} .
\end{aligned}
$$

Заметим, что уравнение (14) можно представить в виде уравнения (3), в котором

$$
\begin{aligned}
& Z_{\alpha \beta}^{1}=\left|\begin{array}{llll}
A_{\beta}^{(21)} & A_{\beta}^{(31)} & 1 & 0 \\
A_{\alpha}^{(21)} & A_{\alpha}^{(31)} & 1 & 0 \\
A_{\beta}^{(22)} & A_{\beta}^{(32)} & 0 & 1 \\
A_{\alpha}^{(22)} & A_{\alpha}^{(32)} & 0 & 1
\end{array}\right|, \quad Z_{\alpha \beta}^{2}=-\left|\begin{array}{cccc}
A_{\beta}^{(11)} & A_{\beta}^{(31)} & 1 & 0 \\
A_{\alpha}^{(11)} & A_{\alpha}^{(31)} & 1 & 0 \\
A_{\beta}^{(12)} & A_{\beta}^{(32)} & 0 & 1 \\
A_{\alpha}^{(12)} & A_{\alpha}^{(32)} & 0 & 1
\end{array}\right| \\
& Z_{\alpha \beta}^{3}=\left|\begin{array}{llll}
A_{\beta}^{(11)} & A_{\beta}^{(21)} & 1 & 0 \\
A_{\alpha}^{(11)} & A_{\alpha}^{(21)} & 1 & 0 \\
A_{\beta}^{(12)} & A_{\beta}^{(22)} & 0 & 1 \\
A_{\alpha}^{(12)} & A_{\alpha}^{(22)} & 0 & 1
\end{array}\right| \\
& T_{\alpha \beta}^{1}=\left|\begin{array}{llll}
A_{\beta}^{(11)} & A_{\beta}^{(21)} & A_{\beta}^{(31)} & 0 \\
A_{\alpha}^{(11)} & A_{\alpha}^{(21)} & A_{\alpha}^{(31)} & 0 \\
A_{\beta}^{(12)} & A_{\beta}^{(22)} & A_{\beta}^{(32)} & 1 \\
A_{\alpha}^{(12)} & A_{\alpha}^{(22)} & A_{\alpha}^{(32)} & 1
\end{array}\right|, \quad T_{\alpha \beta}^{2}=\left|\begin{array}{cccc}
A_{\beta}^{(11)} & A_{\beta}^{(21)} & A_{\beta}^{(31)} & 1 \\
A_{\alpha}^{(11)} & A_{\alpha}^{(21)} & A_{\alpha}^{(31)} & 1 \\
A_{\beta}^{(12)} & A_{\beta}^{(22)} & A_{\beta}^{(32)} & 0 \\
A_{\alpha}^{(12)} & A_{\alpha}^{(22)} & A_{\alpha}^{(32)} & 0
\end{array}\right|,
\end{aligned}
$$




$$
a_{\alpha \gamma \beta}^{(k)}=\left|\begin{array}{llllc}
A_{\gamma}^{(1 k)} & A_{\gamma}^{(2 k)} & A_{\gamma}^{(3 k)} & \delta_{k 1} & \delta_{k 2} \\
A_{\beta}^{(11)} & A_{\beta}^{(21)} & A_{\beta}^{(31)} & 1 & 0 \\
A_{\alpha}^{(11)} & A_{\alpha}^{(21)} & A_{\alpha}^{(31)} & 1 & 0 \\
A_{\beta}^{(12)} & A_{\beta}^{(22)} & A_{\beta}^{(32)} & 0 & 1 \\
A_{\alpha}^{(12)} & A_{\alpha}^{(22)} & A_{\alpha}^{(32)} & 0 & 1
\end{array}\right|, \quad k=1,2 .
$$

Структура уравнения (3) аналогична структуре $(2+1)$-мерного уравнения $n$-волн (1). В частности, оно тождественно равно нулю при $\alpha=\beta$ и допускает редукцию

$$
\begin{gathered}
x_{n}=i \xi_{n}, \quad t_{m}=i \tau_{m}, \quad\left(v^{(l j)}\right)^{+}=v^{(j l)}, \quad i^{2}=-1, \\
n=1,2, \quad m=1,2,3, \quad l, j=0,1,2 .
\end{gathered}
$$

После этой редукции система (3) принимает вид

$$
\begin{gathered}
i \sum_{m=1}^{3} Z_{\alpha \beta}^{(m)}\left(v_{\alpha \beta}^{(l j)}\right)_{\tau_{m}}+i \sum_{n=1}^{2} T_{\alpha \beta}^{(n)}\left(v_{\alpha \beta}^{(l j)}\right)_{\xi_{n}}+\sum_{k=1}^{2} \sum_{\gamma=1}^{N} a_{\alpha \gamma \beta}^{(k)}\left(v_{\alpha \gamma}^{(l k)} v_{\gamma \beta}^{(0 j)}-v_{\alpha \gamma}^{(l 0)} v_{\gamma \beta}^{(k j)}\right)=0, \\
l, j=0,1,2, \quad \alpha, \beta=1, \ldots, N .
\end{gathered}
$$

\section{3. ДОПОЛНИТЕЛЬНЫЕ АЛГЕБРО-ДИФФЕРЕНЦИАЛЬНЫЕ СООТНОШЕНИЯ МЕЖДУ ФУНКЦИЯМИ $v^{(i j)}$}

Согласно разделу 2 недиагональные элементы матричных полей $v^{(i j)}, i, j=0,1,2$, удовлетворяют системе (3). В настоящем разделе мы покажем, что естественным образом возникают дополнительные алгебро-дифференциальные связи между этими матричными полями, включающие их диагональные элементы. Существует три семейства таких связей.

1. К первому семейству относятся уравнения систем (12), (13), в которых $i=j=0$ :

$$
\begin{gathered}
v_{x_{m}}^{(00)}+v^{(0 m)}-v^{(m 0)}-v^{(0 m)} v^{(00)}+v^{(00)} v^{(m 0)}=0 \\
v_{t_{m}}^{(00)}+\sum_{k=1}^{2}\left(v^{(0 k)} A^{(m k)}-A^{(m k)} v^{(k 0)}\right)-\sum_{k=1}^{2} v^{(0 k)} A^{(m k)} v^{(00)}+v^{(00)} \sum_{k=1}^{2} A^{(m k)} v^{(k 0)}=0 .
\end{gathered}
$$

2. Ко второму семейству относятся уравнения систем (12), (13), в которых $j=0$. Исключая поля $v^{(i m 00)}$, представим это семейство в виде

$$
\begin{gathered}
\left|\begin{array}{ccc}
\left(f_{t_{k}}^{(i 0)}\right)_{\alpha \beta} & \left(f_{x_{1}}^{(i 0)}\right)_{\alpha \beta} & \left(f_{x_{2}}^{(i 0)}\right)_{\alpha \beta} \\
A_{\alpha}^{(k 1)} & 1 & 0 \\
A_{\alpha}^{(k 2)} & 0 & 1
\end{array}\right|=0, \quad k=1,2,3, \\
f_{x_{m}}^{(i 0)}:=v_{x_{m}}^{(i 0)}+v^{(i m)}-v^{(i m)} v^{(00)}+v^{(i 0)} v^{(m 0)}, \\
f_{t_{m}}^{(i 0)}:=v_{t_{m}}^{(i 0)}+\sum_{k=1}^{2} v^{(i k)} A^{(m k)}-\sum_{k=1}^{2} v^{(i k)} A^{(m k)} v^{(00)}+v^{(i 0)} \sum_{k=1}^{2} A^{(m k)} v^{(k 0)} .
\end{gathered}
$$


3. K третъему семейству относятся уравнения систем (12), (13), в которых $i=0$. Исключая поля $v^{(00 m j)}$, представим это семейство в виде

$$
\begin{gathered}
\left|\begin{array}{ccc}
\left(f_{t_{k}}^{(0 j)}\right)_{\alpha \beta} & \left(f_{x_{1}}^{(0 j)}\right)_{\alpha \beta} & \left(f_{x_{2}}^{(0 j)}\right)_{\alpha \beta} \\
A_{\beta}^{(k 1)} & 1 & 0 \\
A_{\beta}^{(k 2)} & 0 & 1
\end{array}\right|=0, \quad k=1,2,3, \\
f_{x_{m}}^{(0 j)}:=v_{x_{m}}^{(0 j)}-v^{(m j)}-v^{(0 m)} v^{(0 j)}+v^{(00)} v^{(m j)}, \\
f_{t_{m}}^{(0 j)}:=v_{t_{m}}^{(0 j)}-\sum_{k=1}^{2} A^{(m k)} v^{(k j)}-\sum_{k=1}^{2} v^{(0 k)} A^{(m k)} v^{(0 j)}+v^{(00)} \sum_{k=1}^{2} A^{(m k)} v^{(k j)} .
\end{gathered}
$$

Все соотношения (21)-(23) имеют диагональные части и включают диагональные элементы всех матричных полей $v^{(i j)}, i, j=0,1,2$. Однако следует подчеркнуть, что наличие этих связей не разрушает полной интегрируемости системы (3). Мы поясним это утверждение в разделе 4.

\section{4. ПРОСТРАНСТВО РЕШЕНИЙ СИСТЕМЫ (3)}

Прежде всего в данной главе мы покажем полную интегрируемость системы (3). Для этого снова обратимся к спектральному представлению и выведем спектральные функции $\Psi$ и матричные поля $v^{(i j)}$ в явном виде.

4.1. Вывод явных формул для $\Psi$ и $v^{(i j)}$. Чтобы получить $\Psi$ в явном виде, следует обратить оператор $R$, т. е. решить интегральное уравнение $(7)$ относительно $\Psi$. Важно то, что $\Psi$ можно построить в явном виде с точностью до обратимости интегрального оператора $R_{0}$, не зависящего от переменных $x$ и $t$. Подставляя $R$ из (5), перепишем уравнение (7) в виде

$$
\int \widehat{R}_{0}(\lambda, \nu) \Psi(\nu, \mu) d \Omega(\nu)+r(\lambda) \chi(\mu)=\delta(\lambda-\mu),
$$

где

$$
\begin{gathered}
\widehat{R}_{0}(\lambda, \nu)=\exp \left(\sum_{j=1}^{D} s^{(j)}(\lambda) x_{j}+\sum_{k} S^{(k)}(\lambda) t_{k}\right) R_{0}(\lambda, \mu) \times \\
\times \exp \left(-\sum_{j=1}^{D} s^{(j)}(\mu) x_{j}-\sum_{k} S^{(k)}(\lambda) t_{k}\right), \\
\chi(\mu)=\int q(\nu) \Psi(\nu, \mu) d \Omega(\nu) .
\end{gathered}
$$

Предполагая обратимость $R_{0}(\lambda, \mu)$, запишем выражение

$$
\begin{aligned}
\widehat{R}_{0}^{-1}(\lambda, \mu)= & \exp \left(\sum_{j=1}^{D} s^{(j)}(\lambda) x_{j}+\sum_{k} S^{(k)}(\lambda) t_{k}\right) R_{0}^{-1}(\lambda, \mu) \times \\
& \times \exp \left(-\sum_{j=1}^{D} s^{(j)}(\mu) x_{j}-\sum_{k} S^{(k)}(\mu) t_{k}\right),
\end{aligned}
$$


где $R_{0}(\lambda, \nu) * R_{0}^{-1}(\nu, \mu)=\delta(\lambda-\mu)$. Тогда, применяя $\widehat{R}_{0}^{-1} *$, найдем $\Psi$ в виде

$$
\Psi=\widehat{R}_{0}^{-1}-\widehat{R}_{0}^{-1} * r \chi
$$

Чтобы найти функцию $\chi$ в правой части этого уравнения, применим оператор $q *$ к уравнению (28) и решим полученное уравнение относительно $\chi$. Получаем, что

$$
\chi=\left(1+w^{(00)}\right) q * \widehat{R}_{0}^{-1},
$$

где используются следующие обозначения:

$$
w^{(i j)}=\left(q s^{(i)}\right) * \widehat{R}_{0}^{-1} *\left(s^{(j)} r\right), \quad i, j=0,1, \ldots, D, \quad s^{(0)}=I_{N} .
$$

Применяя операторы $\left(q s^{(i)}\right) *$ и $*\left(s^{(j)} r\right)$ к уравнению $(28)$, приходим к выражению

$$
v^{(i j)}=w^{(i j)}-w^{(i 0)}\left(I+w^{(00)}\right)^{-1} w^{(0 j)} .
$$

Очевидно, каждая из функций $w^{(i j)}$ является решением соответствующего линейного уравнения в частных производных (4). Решения $v^{(i j)}$ не имеют особенностей в том случае, если $\operatorname{det}\left(I+w^{(00)}\right) \neq 0$ для всех $x_{i}$ и $t_{i}$ внутри интересующей нас области.

Вывод о полной интегрируемости системы (3) следует из того факта, что функция $R(25)$ произвольным образом зависит от $2 D$ независимых функций спектральных параметров $s^{(i)}(\lambda)$ и $s^{(i)}(\mu)$, что определяет произвольные функции от $2 D$ независимых переменных $x_{i}$ и $t_{i}$. Это требование необходимо для полной интегрируемости системы $(2 D+1)$-мерных уравнений в частных производных. Очевидно, число этих произвольных матричных функций от переменных $(x, t)$ совпадает с числом полей $v^{(i j)}$ в системе (3). Действительно, эти произвольные функции есть не что иное, как поля $w^{(i j)}(30)$. По построению это независимые функции с произвольной зависимостью от $2 D$ переменных $x_{i}$ и $t_{i}$.

Кроме того, чтобы удовлетворить редукции (19), наложим следующие ограничения на функции спектральных параметров $R_{0}$ и $s$ :

$$
R_{0}(\lambda, \mu)=R_{0}^{+}\left(\mu^{*}, \lambda^{*}\right), \quad q(\lambda)=r^{*}\left(\lambda^{*}\right), \quad s^{(n)}(\lambda)=\left(s^{(n)}\right)^{*}\left(\lambda^{*}\right),
$$

где звездочка означает комплексное сопряжение и учтена диагональность функций $r, q$ и $s^{(n)}$.

4.2. Частные решения. В этом пункте рассматриваются частные решения $v^{(i j)}$ при наложении редукции (19) (т. е. решения уравнения (20)), построенные с помощью дискретных спектральных параметров $\lambda, \mu \in\left\{z_{1}, z_{2}, \ldots, z_{M}\right\}$, где $z_{i}$ - действительные числа. В качестве примера возьмем следующие функции $R_{0}, r, q$ и $s^{(n)}$, удовлетворяющие редукции (32):

$$
\begin{gathered}
\left(R_{0}\left(z_{j}, z_{k}\right)\right)_{\alpha \beta}=\delta_{\alpha \beta} \delta_{j k}+\alpha^{z_{j}} \beta^{z_{k}}\left(1-\delta_{j k}\right), \quad s_{\alpha}^{(n)}\left(z_{j}\right)=\left(\alpha z_{j}\right)^{n}, \\
A_{\alpha}^{(m n)}=(\alpha+n-1)^{m}, \quad r_{\alpha}\left(z_{j}\right)=q_{\alpha}\left(z_{j}\right)=\alpha^{z_{j}}, \\
n=1,2, \quad m=1,2,3, \quad j, k=1, \ldots, M, \quad \alpha, \beta=1, \ldots, N,
\end{gathered}
$$


где $\delta_{i j}$ - символ Кронекера. В этом случае поля $v^{(i j)}$ задаются формулой $(31)$, в которой функции $w^{(i j)}$ определяются выражениями (30) и (25):

$$
w^{(i j)}=\sum_{i, j=1}^{M} \alpha^{z_{i}}\left(\alpha z_{i}\right)^{n} e^{K_{\alpha}\left(x, t, z_{i}\right)-K_{\beta}\left(x, t, z_{j}\right)}\left(R_{0}^{-1}\right)_{\alpha \beta}\left(z_{i}, z_{j}\right)\left(\beta z_{j}\right)^{m} \beta^{z_{j}},
$$

где

$$
K_{\alpha}\left(x, t, z_{i}\right)=\sum_{j=1}^{2}\left(\alpha z_{i}\right)^{j} x_{j}+\sum_{l=1}^{3} \sum_{k=1}^{2}(\alpha+k-1)^{l}\left(\alpha z_{i}\right)^{k} t_{l}
$$

а $R_{0}^{-1}$ - оператор, обратный к $R_{0}$ :

$$
\sum_{l=1}^{M} \sum_{\gamma=1}^{N}\left(\delta_{\alpha \gamma} \delta_{i l}+\alpha^{z_{i}} \gamma^{z_{l}}\left(1-\delta_{i l}\right)\right)\left(R_{0}^{-1}\right)_{\gamma \beta}\left(z_{l}, z_{j}\right)=\delta_{\alpha \beta} \delta_{i j}
$$

На рис. 1 изображены модули некоторых матричных элементов полей $v^{(i j)}$ для случая $M=2$ и $N=4$ на плоскости $\left(\xi_{1}, \xi_{2}\right)$ при $\tau_{i}=i$.

Как видно, все элементы матриц $v^{(i j)}$ можно разделить на три семейства. Модули матричных элементов первого семейства (элементов матриц $v^{(00)}$ и $v^{(10)}$, см. рис. 1а) имеют высокие пики, регулярно расположенные на плоскости $\left(\xi_{1}, \xi_{2}\right)$. Модули матричных элементов второго семейства (элементов матрицы $v^{(01)}$, см. рис. 1б) имеют пики, расположенные на осциллирующей платформе. Наконец, модули матричных элементов третьего семейства (элементов матрицы $v^{(11)}$, см. рис. 1в) не имеют острых пиков. Следовательно, такого рода уравнения типа $n$-волн могут быть связаны с формированием квазипериодических решений в виде лампов большой амплитуды.

\section{5. ВЫВОДЫ}

Построена полностью интегрируемая линеаризуемая версия пятимерного уравнения типа $n$-волн для матричных полей $v^{(i j)}, i, j=0,1,2$. Его обобщение на случай размерности $2 D+1, D>2$, очевидно. Показано, что элементы матричных полей $v^{(i j)}$ имеют разные формы. Некоторые из них имеют вид квазипериодически расположенных острых пиков и, следовательно, могут быть связаны с формированием волн-убийц.

Интересно отметить, что для вывода уравнения (3) была введена вспомогательная спектральная функция $\Psi$, поэтому поля $v^{(i j)}$ приобретают спектральное представление по аналогии с полями нелинейных уравнений в частных производных, интегрируемых с помощью МОЗР. Таким образом, метод одевания появляется здесь как средство объединения нелинейных уравнений в частных производных, интегрируемых различными методами. Аналогичное явление наблюдалось ранее в работах [15], [16], в которых рассмотрены комбинации нелинейных уравнений в частных производных, интегрируемых различными способами, такими как МОЗР, линеаризация подстановкой Хопфа, метод характеристик. 


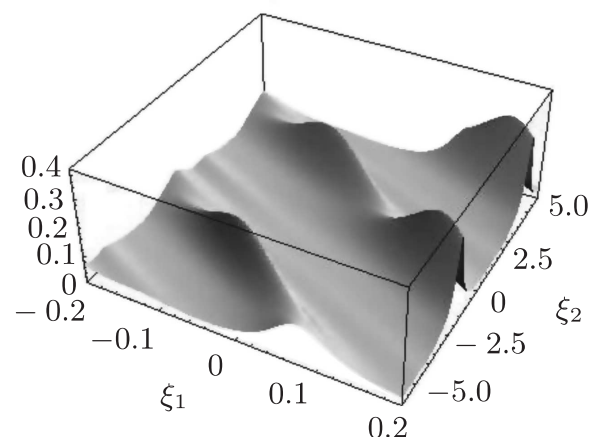

a

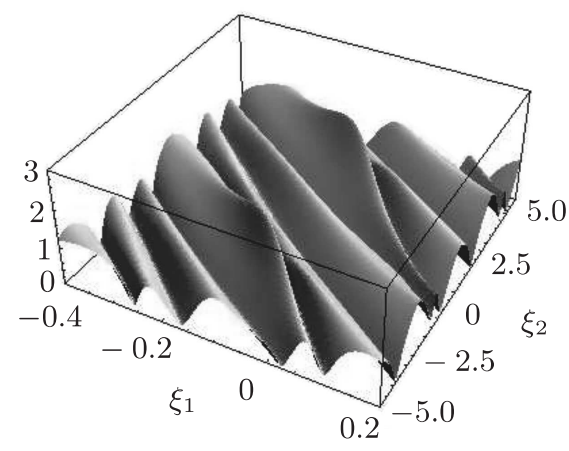

б

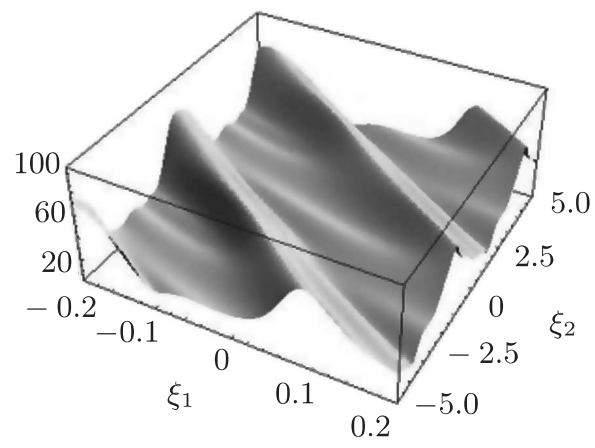

B

Рис. 1. Модули некоторых матричных элементов частного решения уравнения (20) при $\tau_{i}=i, i=1,2,3$. Показаны три типичных случая: модули матричных элементов полей $v^{(00)}$ и $v^{(10)}$ имеют множество направленных вверх пиков, распределенных на плоскости $\left(\xi_{1}, \xi_{2}\right)$, в качестве примера приведен модуль $\left|v_{34}^{(00)}\right|\left(\right.$ a); модули матричных элементов поля $v^{(01)}$ имеют множество направленных вверх пиков, распределенных на осциллирующей основе, в качестве примера приведен модуль $\left|v_{14}^{(01)}\right|$ (б); модули матричных элементов поля $v^{(11)}$ имеют осциллирующий вид без пиков, в качестве примера показан модуль $\left|v_{34}^{(11)}\right|($ в).

Конечно, вместо ядра $\widehat{R}_{0}$ симметричного вида $(25)$ можно использовать его несимметричную версию

$$
\begin{aligned}
\widehat{R}_{0}(\lambda, \nu)= & \exp \left(\sum_{j=1}^{D} s^{(j)}(\lambda) x_{j}+\sum_{k} S^{(k)}(\lambda) t_{k}\right) R_{0}(\lambda, \mu) \times \\
& \times \exp \left(\sum_{j=1}^{D} p^{(j)}(\mu) x_{j}+\sum_{k} P^{(k)}(\lambda) t_{k}\right) .
\end{aligned}
$$

Тогда полученные нелинейные уравнения в частных производных содержат также и диагональную часть. 


\section{Список литературы}

[1] D. J. Kaup, "A method for solving the separable initial-value problem of the full threedimensional three-wave interaction", Stud. Appl. Math., 62:75-83 (1980).

[2] D. J. Kaup, "The inverse scattering solution for the full three-dimensional three-wave resonant interaction", Phys. D, 1:45-67 (1980).

[3] C.S. Gardner, J. M. Green, M.D. Kruskal, R. M. Miura, "Method for solving the Korteweg-deVries equation", Phys. Rev. Lett., 19:19 (1967), 1095-1097.

[4] В.Е. Захаров, С.В. Манаков, С.П. Новиков, Л. П. Питаевский, Теория солитонов. Метод обратной задачи, Наука, М., 1980.

[5] M. J. Ablowitz, P. C. Clarkson, Solitons, Nonlinear Evolution Equations and Inverse Scattering, London Mathematical Society Lecture Note Series, 149, Cambridge Univ. Press, Cambridge, 1991.

[6] А.Б. Шабат, "Об уравнении Кортевега-де Фриза”, Докл. АН CCCP, 211:6 (1973), $1310-1313$.

[7] В. Е. Захаров, А. Б. Шабат, “Схема интегрирования нелинейных уравнений математической физики методом обратной задачи рассеяния. I", Функи. анал. и его прил., 8:3 (1974), 43-53.

[8] В. Е. Захаров, А. Б. Шабат, "Интегрирование нелинейных уравнений математической физики методом обратной задачи рассеяния. II", Функи. анал. и его прил., 13:3 (1979), $13-22$.

[9] В.Е. Захаров, С. В. Манаков, “Построение многомерных нелинейных интегрируемых систем и их решений”, Функи. анал. и его прил., 19:2 (1985), 11-25.

[10] B.G. Konopelchenko, Solitons in Multidimensions. Inverse Spectral Transform Method, World Sci., Singapore, 1993.

[11] A.I. Zenchuk, "On integration of a multidimensional version of $n$-wave type equation", J. Math. Phys., 55:12 (2014), 121505, 30 pp.

[12] V. E. Zakharov (ed.), What is Integrability?, Springer, Berlin, 1991.

[13] F. Calogero, Ji Xiaoda, "C-integrable nonlinear partial differentiation equations", J. Math. Phys., 32:4 (1991), 875-887.

[14] F. Calogero, Ji Xiaoda, "C-integrable nonlinear PDEs. II", J. Math. Phys., 32:10 (1991), $2703-2717$.

[15] A. I. Zenchuk, "A unified dressing method for $C$ - and $S$-integrable hierarchies; the particular example of a $(3+1)$-dimensional $n$-wave equation", J. Phys. A: Math. Gen., 37:25 (2004), $6557-6571$.

[16] A. I. Zenchuk, "On the relationship between nonlinear equations integrable by the method of characteristics and equations associated with commuting vector fields", J. Math. Phys., 50:6 (2009), 063505, 18 pp.

Поступила в редакцию 1.04.2016, после доработки 15.04.2016 